\title{
Article
}

\section{On-Axis Resolution of a Circular Aperture}

\author{
Maria Antonia Maisto
}

check for updates

Citation: Maisto, M.A. On-Axis Resolution of a Circular Aperture. Electronics 2021, 10, 729. https:// doi.org/10.3390/electronics10060729

Academic Editor: Francisco Falcone

Received: 18 February 2021

Accepted: 16 March 2021

Published: 19 March 2021

Publisher's Note: MDPI stays neutral with regard to jurisdictional claims in published maps and institutional affiliations.

Copyright: (C) 2021 by the author. Licensee MDPI, Basel, Switzerland. This article is an open access article distributed under the terms and conditions of the Creative Commons Attribution (CC BY) license (https:// creativecommons.org/licenses/by/ $4.0 /)$.
Department of Engineering, University of Campania Luigi Vanvitelli, Via Roma 29, 81031 Aversa, Italy; mariaantonia.maisto@unicampania.it; Tel.: +39-0815010335

\begin{abstract}
In this paper, the problem to estimate the on-axis resolution in creating a desired field profile by radiation of an aperture $A$ is addressed. The latter applies in both diffractive optics and antenna synthesis. This is because the 'aperture theory', $A$ can schematize a source, for example, an antenna or a lens illuminated by an incident field radiating a significant field only on the same aperture. The analysis refers to a customary axicon geometry consisting of a circle aperture transverse to the observation domain. The aim was to find a resolution formula allowing to highlight the impact of aperture geometrical parameters for configurations that are below the Fresnel approximation. The results show that the aperture cannot approximate the target field with the same level of accuracy along with the observation domain. In particular, near the aperture, smaller details can be retrieved and as the distance increases this ability degrades.
\end{abstract}

Keywords: resolution estimation; field synthesis; radiation

\section{Introduction}

In this paper, the problem of creating a desired field distribution by the radiation of an aperture $A$ is considered. The latter applies in both diffractive optics and antenna synthesis. This is because the 'aperture theory', $A$ can schematize a source, for example, an antenna or a lens illuminated by an incident field radiating a significant field only on the same aperture. In this framework, it then is important to know if the aperture is able to create any desired field profile, and how accurately.

The resolution is a figure of merit that quantifies the ability to create details of such a field profile. Unfortunately, this parameter is limited and mainly depends on the geometrical features of the aperture $A$ [1]. Accordingly, in this paper, the focus is to estimate the above-mentioned resolution limits in terms of aperture geometrical parameters. Such a purpose has been addressed in many recent papers in the framework of inverse source [2-5]. There, the problem is to reconstruct an unknown current from the measure of its radiated field. Accordingly, resolution is estimated when source and observation domain are parallel strips [2], orthogonal strips [3,4] and in presence of inhomogeneous medium [5]. Note that the point, here, is not to reconstruct the aperture field from the given field distribution, but rather to explore the aperture capability of radiating that fixed field. However, the problems are related and in both, the resolution is limited.

The formulation proposed in the present paper is more similar to the one provided in [6]. In that paper, the above-mentioned resolution limit is studied by the method of communication modes previously employed in information theory [7]. The latter [7] is a physically insightful method for (back-)propagating waves between two volumes of arbitrary shape and for analyzing the information content transmitted between them. Such an approach is based on the singular-value decomposition (SVD) of the operator linking the waves over the two volumes, also known as radiation operator. Within this framework, estimating the significant singular values means knowing the maximal number of communication modes that are well connected. When the evanescent contribution in the radiation operator is neglected, the smoothness of its kernel function increases [8] and the singular values present an abrupt and exponentially quick decay beyond a certain critical 
index. This practically entails that the number of well-connected communication modes is always bounded and dependent on the geometrical parameters of the two volumes. Such a number is known as Number of Degree of Freedom (NDF) [9] and it is linked to resolution because it gives a concise picture of which class of field profile the aperture can create $[10,11]$. In fact, in [6], it is shown that any field created by an aperture can consist of no finer details than those contained in the singular functions spanning the closure of the Range of radiation operator $v_{n}$ with $n \leq N D F$. Accordingly, the boundedness of NDF entails that the resolution is finite and its expression depends mainly on the geometrical parameters of the aperture.

In this paper, we aim to estimate the above-mentioned expression for the case of a circular aperture and an observation domain located on its axis. For this reason, we refer to it as on-axis resolution. Note that this a customary axicon geometry which has already been studied in [12-15]. However, it must be pointed out that although the references [12-14] concern the geometry addressed in this paper, their aim was different from the one proposed here. In fact, in [12-14], the focus is not on the resolution limits of the aperture, rather the aim was to study the diffraction by a circular aperture of a plane wave or other types of prescribed focused beam. In fact, in this paper, we do not refer to any particular incidence field and we consider the aperture as the result of the interaction between a lens and an incidence field. Such a problem is relevant in lens synthesis and the results obtained in this manuscript suggest how the aperture geometrical parameters must be set in order to obtain the desired resolution. The only reference which addresses such an aim is [15] where by exploiting the approach developed in [4], the singular values decomposition of the radiation operator is evaluated in closed form. According to such evaluation, an expression for the resolution is given in terms of geometrical parameters. However, such expression is derived under the so-called Fresnel approximation, which is known to put serious restrictions on the distance between aperture and observation domain.

Therefore, we expand the study developed in [15] in order to cover configurations that are not within the Fresnel approximation. This means that we allow the observation domain to be closer to the aperture. However, we assume that the latter is not close enough to make the evanescent contributions relevant. Accordingly, the considered configuration can be addressed as being in the near non-reactive zone. It is worth noting that such a zone is the same to which the pseudo-Fresnel approximation provided in [14] applies.

The resolution is identified as the distance between the maximum and the first zero of the intensity point-spread function (psf) [1]. Since the psf can be expressed in terms of the NDF and the singular functions $v_{n}$, the resolution estimation requires the knowledge of the SVD in closed form.

For a such configuration, unlike the Far [16] and Fresnel zone [15], the singular values decomposition cannot be derived in closed form so an alternative approach to evaluating the point-spread function is proposed. This is based on the observation that to find the field over the aperture ensuring a target field profile on the observation domain requires solving an inverse problem. As shown in [2,3], this can be achieved by the weighted adjoint inversion method. Such a method consists of approximating the inverse of the radiation operator with its adjoint after performing a pre-filtering step over the data. Such prefiltering type is established by choosing the weighting function in order to obtain a focusing psf. The weighting function can be evaluated easily when the operator is Fourier integral one. Unfortunately, this is not the actual case. However, some phase stationary arguments [2,3] can be exploited to re-write the operator as a Fourier integral one. The resulting approximation of the psf is in agreement with the one derived by the SVD, in particular in its main lobe. Accordingly, an estimation for the resolution is given in terms of the configuration parameters and the latter matches the one derived in [15] when the Fresnel approximation applies. 


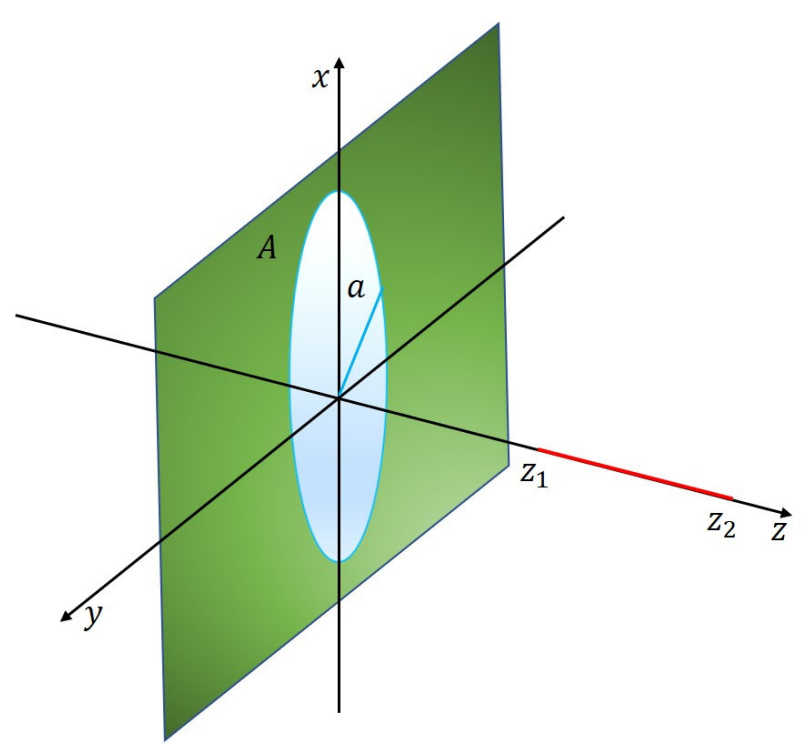

Figure 1. Geometry of the problem.

\section{Mathematical Formulation}

Consider the circular aperture $A$ sketched in Figure 1 consisting of a circle of radius $a$ and located at $z=0$. In the framework of 'aperture theory', such an aperture can schematize a source, for example an antenna or a lens illuminated by an incident field, located in the half-space $z<0$ and radiating a significant field only on $A$. Let $\underline{E}_{a p}$ be the field over such an aperture, directed along the $x-y$ plane and $\underline{E}$ the field radiated by it. If we omit an unessential scalar factor, $\underline{E}$ is linked to $\underline{E}_{a p}$ by the following vectorial equation

$$
\left[\begin{array}{c}
E_{x}\left(\mathbf{r}_{o}\right) \\
E_{y}\left(\mathbf{r}_{o}\right) \\
E_{z}\left(\mathbf{r}_{o}\right)
\end{array}\right]=\int_{A}\left[\begin{array}{cc}
-\frac{\partial g}{\partial z_{o}}\left(\mathbf{r}_{o}, \mathbf{r}\right) & 0 \\
0 & \frac{\partial g}{\partial z_{o}}\left(\mathbf{r}_{o}, \mathbf{r}\right) \\
\frac{\partial g}{\partial x_{o}}\left(\mathbf{r}_{o}, \mathbf{r}\right) & -\frac{\partial g}{\partial y_{o}}\left(\mathbf{r}_{o}, \mathbf{r}\right)
\end{array}\right] \cdot \mathbf{E}_{a p}(\mathbf{r}) d \mathbf{r}
$$

where

$$
g\left(\mathbf{r}_{o}, \mathbf{r}\right)=-\frac{e^{-j k\left|\mathbf{r}_{o}-\mathbf{r}\right|}}{4 \pi\left|\mathbf{r}_{o}-\mathbf{r}\right|}
$$

is the 3D scalar Green function and $k$ is the free-space wave number. In addition, $\mathbf{r}_{o}=$ $\left(x_{0}, y_{0}, z_{0}\right)$ denotes the observation point and $\left|\mathbf{r}_{o}-\mathbf{r}\right|$ the distance between the latter and a point on the aperture $\mathbf{r}=(x, y, 0)$. Suppose that only the components of $\underline{E}$ in $x-y$ plane are observed on the $z$ axis within the observation region ranging from $z_{1}$ to $z_{2}$. Then, by expressing the integral in $\mathbf{r}$ in terms of cylindrical coordinates $(\rho \cos (\theta), \rho \sin (\theta), 0),(1)$ becomes

$$
\left[\begin{array}{c}
E_{x}(z) \\
E_{y}(z)
\end{array}\right]=\int_{A}\left[\begin{array}{cc}
-\frac{\partial g}{\partial z_{o}}(z, \rho) & 0 \\
0 & \frac{\partial g}{\partial z_{o}}(z, \rho)
\end{array}\right] \cdot \mathbf{E}_{a p}(\rho, \theta) \rho d \theta d \rho
$$

It is evident from (3) that such a vectorial equation splits into two completely equivalent scalar ones; accordingly, in this paper, we limit to analyze this common scalar problem.

$$
E(z)=-\int_{0}^{a} G(z, \rho) e^{-j k R} \bar{E}_{a p}(\rho) d \rho
$$

where $G(z, \rho)=\frac{z \rho}{R^{2}}\left[j k+\frac{1}{R}\right]$ and $R=\sqrt{\rho^{2}+z^{2}}$. Note that for brevity we suppressed the field component indexes. Since the kernel function of integral equation does not 
depend on $\theta$ variable, $\bar{E}_{a p}(\rho)$ is defined as the mean value along $\theta$ of $E_{a p}(\rho, \theta)$, that is, $\bar{E}_{a p}(\rho)=\int_{0}^{2 \pi} E_{a p}(\rho, \theta) d \theta$. In operator form, the Equation (4) becomes

$$
\mathcal{A}: \bar{E}_{a p} \in L^{2}[0, a] \rightarrow E \in L^{2}\left[z_{1}, z_{2}\right]
$$

in which $\mathcal{A}$ is a linear integral operator acting between the square integrable sets $L^{2}[0, a]$ and $L^{2}\left[z_{1}, z_{2}\right]$.

Note that the mathematical model in (3) is equivalent to one derived in [17] where the electromagnetic description of a refractive axicon lens is provided. In particular, reference [17] shows that when the lens is illuminated normally by a plane wave uniformly polarized $E_{i n c}=E_{0} e^{-j k z} \hat{u}$ (with $\hat{u}$ indicating the state of polarization), the field on the equivalent aperture $\underline{E}_{a p}(\rho)$ is dependent only on $\rho$ variable and is related to the incidence field by a scalar transformation function $\tau(\rho)$. Such a transformation $\tau(\rho)$ describes the features of the lens and its dependence on the $\rho$ variable is of exponential type. For such a structure, the image segment is formed on the $z$ axis within the observation region ranging from $z_{1}$ to $z_{2}$. Accordingly, the result provided in this paper applies also to this structure.

\subsection{Point-Spread Function}

Assuming a desired target field profile $E_{o}$ on the observation interval $\left[z_{1}, z_{2}\right]$, its best possible approximation $E_{i}$ that the aperture shown in Figure 1 creates, can be estimated as

$$
E_{i}(z)=\int_{z_{1}}^{z_{2}} p s f\left(z, z^{\prime}\right) E_{o}\left(z^{\prime}\right) d z^{\prime}
$$

where $\operatorname{ps} f\left(z, z^{\prime}\right)$ is the point-spread function. This equation makes clear that $E_{i}$ is a filtered version of $E_{o}$ and the goodness of such filtering depends on the aperture.

Since the compactness of $\mathcal{A}$, its singular system $\left\{\sigma_{n}, u_{n}, v_{n}\right\}_{n=0}^{\infty}$ can be introduced. In particular, the following equations hold

$$
\begin{gathered}
\sigma_{n} v_{n}=\mathcal{A} u_{n} \\
\sigma_{n} u_{n}=\mathcal{A}^{\dagger} v_{n}
\end{gathered}
$$

with $\mathcal{A}^{\dagger}$ being the adjoint operator of $\mathcal{A}$. The $\sigma_{n}$ are the singular values ordered in a nonincreasing sequence and repeated according to their multiplicity, and $u_{n}$ and $v_{n}$ are the singular functions, orthonormal bases for the orthogonal complement of the null space of $\mathcal{A}$ and the closure of the Range of $\mathcal{A}$, respectively. According to the theory of the communication modes, an expression for the $p s f$ can be given in terms of the $v_{n}$

$$
\operatorname{psf}\left(z, z^{\prime}\right)=\sum_{n=0}^{N_{\epsilon}} v_{n}^{*}\left(z^{\prime}\right) v_{n}(z)
$$

where the symbol * is the conjugate and $N_{\epsilon}$ represents the number of "significant" singular values, that is the $\sigma_{n}$ greater than a threshold linked to the desired approximation error.

The compactness of $\mathcal{A}$ entails that the $\sigma_{n}$ goes to zero. In particular, as can be appreciated from Figure 2, when $z>\lambda$, with $\lambda$ the wavelength, the evanescent contribution can be neglected and the singular values after a starting dynamic decay exponentially in correspondence with the index $N$. Such a number is known as the Number of Degree of Freedom and gives a measure of the maximal number of communication modes that are "significantly" connected. Hence, according to the theory of communication mode, only a finite number of modes $N$ are required to represent the resulting field $E_{i}$. The resolution limit of such an aperture has its mathematical foundation in this equation: indeed $E_{i}$ can consist of no finer details (higher spatial frequencies) than those contained in $v_{n}$ with $n \leq N$. In order to find the resolution limit, an explicit expression for the $p s f$ in terms of configuration parameters should be derived. Unfortunately, the singular system of $\mathcal{A}$ is not known in closed form, so we need to find an alternative approach that allows us to obtain an approximation of (8). 

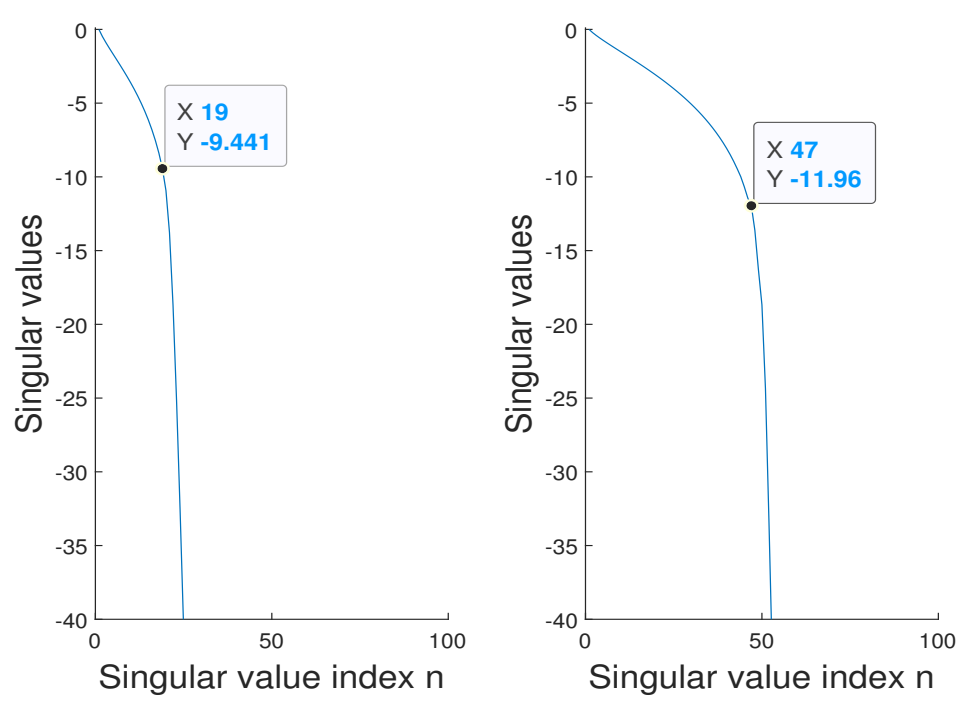

Figure 2. Normalized singular value behavior of the operator $\mathcal{A}$ in $\mathrm{db}$ for $z_{1}=2 \lambda$ and $z_{2}=50 \lambda$. On the left, $a=30 \lambda$, on the right $a=15 \times 10^{3} \lambda$.

In order to address a such purpose, we consider the aperture field $\bar{E}_{a p}$ that allows to create $E_{i}$. Its expression is given by

$$
\bar{E}_{a p}(\rho)=\sum_{n=0}^{N} \frac{<E_{o}, v_{n}>}{\sigma_{n}} u_{n}(\rho)
$$

Note that (9) is the solution of the inverse problem $\mathcal{A} \bar{E}_{a p}=E_{i}$ obtained by exploiting the truncated singular value decomposition (TSVD) as a regularization scheme. This is evident by applying $\mathcal{A}$ on (9) and exploiting the first of (7).

Accordingly, the matter of the fact is to find an alternative approach that allows obtaining (9) with the same level of accuracy. As shown in [2,3], this can be reached by the weighted adjoint inversion method. It is a filtered backpropagation [18] and as the TSVD belongs to the class of Tikhonov regularization scheme [19]. According to such an approach, the $\bar{E}_{a p}$ can be expressed as

$$
\bar{E}_{a p}=\mathcal{A}_{h}^{\dagger}\left(E_{o}\right)
$$

where

$$
\begin{gathered}
\mathcal{A}_{h}^{+}: E \in L^{2}\left[z_{1}, z_{2}\right] \rightarrow \\
\left.\bar{E}_{a p}=-\int_{z_{1}}^{z_{2}} h(\rho, z) G^{*}(\rho, z)\right) e^{j k R} E(z) d z \in L^{2}[0, a]
\end{gathered}
$$

with $h(\rho, z)$ as a weighting function. Hence, by applying $\mathcal{A}$ on (10), we obtain

$$
E_{i}(z)=\int_{z_{1}}^{z_{2}} p s f_{h}\left(z, z^{\prime}\right) E_{o}\left(z^{\prime}\right) d z^{\prime}
$$

where the resulting $p s f_{h}\left(z, z^{\prime}\right)$ is given by

$$
\operatorname{ps} f_{h}\left(z, z^{\prime}\right)=\int_{0}^{a} h\left(\rho, z^{\prime}\right) G^{*}\left(\rho, z^{\prime}\right) G(\rho, z) e^{-j\left[\phi(\rho, z)-\phi\left(\rho, z^{\prime}\right)\right]}() d \rho
$$

with $\phi=k R$. It is evident that in order to obtain the best possible approximation of $E_{o}$, $h$ should be chosen in order to ensure that the $p s f_{h}\left(z, z^{\prime}\right)$ is as close as possible to a delta function. 


\subsection{Estimation of $p s f_{h}$}

In order to derive the function $h$, we need to re-express (13) as a Fourier integral operator. To address such a purpose, we introduce the following spectral variable defined as

$$
w\left(z, z^{\prime}, \rho\right)=\left.\int_{0}^{1} \frac{\partial \phi(\rho, p)}{\partial p}\right|_{p=z^{\prime}+v\left(z-z^{\prime}\right)} d v
$$

such as

$$
\phi(\rho, z)-\phi\left(\rho, z^{\prime}\right)=w\left(z, z^{\prime}, \rho\right)\left(z-z^{\prime}\right)
$$

The function $w\left(z, z^{\prime}, \rho\right)$ is continuous and monotonic (hence, invertible) $\forall z, z^{\prime}$. Accordingly, integration in $\rho$ can be replaced with integration in $w$, so that (13) re-writes as

$$
p s f_{h}\left(z, z^{\prime}\right)=\int_{w\left(z, z^{\prime}, 0\right)}^{w\left(z, z^{\prime}, a\right)} h\left(\rho, z^{\prime}\right) G^{*}\left(\rho, z^{\prime}\right) G(\rho, z) e^{-j\left[w\left(z, z^{\prime}, \rho\right)\left(z-z^{\prime}\right)\right]} \frac{\partial \rho}{\partial w} d w
$$

Then by centering the integral in $w$ and setting

$$
\Delta w\left(z, z^{\prime}\right)=\frac{w\left(z, z^{\prime}, a\right)-w\left(z, z^{\prime}, 0\right)}{2}
$$

and

$$
w_{m}\left(z, z^{\prime}\right)=\frac{w\left(z, z^{\prime}, a\right)+w\left(z, z^{\prime}, 0\right)}{2},
$$

one obtains the following expression for (16)

$$
p s f_{h}\left(z, z^{\prime}\right)=e^{-j\left[\gamma(z)-\gamma\left(z^{\prime}\right)\right]} \int_{-\Delta w\left(z, z^{\prime}\right)}^{\Delta w\left(z, z^{\prime}\right)} h\left(\rho, z^{\prime}\right) G^{*}\left(\rho, z^{\prime}\right) G(\rho, z) \frac{\partial \rho}{\partial w} e^{-j\left[w\left(z, z^{\prime}, \rho\right)\left(z-z^{\prime}\right)\right]} d w
$$

where the following identity has been observed

$$
w_{m}\left(z-z^{\prime}\right)=\gamma(z)-\gamma\left(z^{\prime}\right)
$$

with $\gamma(z)=\frac{k}{2}[R(a, z)+R(0, z)]$.

Under the hypothesis that $z>\lambda, G(\rho, z)$ can be approximated with $\frac{z \rho}{R^{2}} j k$ (evanescent contribution is neglected) and the amplitude term in (19) is a constant sign function and slowly variant. This entails that the leading order contribution in (19) occurs for $z-z^{\prime}=0$ [20]. Accordingly, the amplitude factor can be approximated by its value assumed for $z=z^{\prime}$ (that is $\left.G(\rho, z) \frac{\partial \rho}{\partial w} \approx G\left(\rho, z^{\prime}\right) \frac{\partial \rho}{\partial w}\right|_{z=z^{\prime}}$ ) and the resulting point spread function is

$$
p s f_{h}\left(z, z^{\prime}\right)=e^{-j\left[\gamma(z)-\gamma\left(z^{\prime}\right)\right]} \int_{-\Delta w\left(z, z^{\prime}\right)}^{\Delta w\left(z, z^{\prime}\right)} K\left(w, z^{\prime}\right) e^{-j\left[w\left(z, z^{\prime}, \rho\right)\left(z-z^{\prime}\right)\right]} d w
$$

with $K\left(w, z^{\prime}\right)=\left.h\left(\rho, z^{\prime}\right)\left|G\left(\rho, z^{\prime}\right)\right|^{2} \frac{\partial \rho}{\partial w}\right|_{z=z^{\prime}}$. As can be seen, (21) expresses the point-spread function in terms of both $z$ and $z^{\prime}$ as a spatially variant band limited function [21-23], that is, a function whose bandwidth $\left(\Delta w\left(z, z^{\prime}\right)\right)$ depends on both $z$ and $z^{\prime}$. This $p s f$ behavior is a distinctive feature of near-zone configuration which has been already observed in literature $[2,3,24,25]$ and leads to spatially varying resolution.

From (21), it is evident that the best we can do is to make $p s f_{h}$ as similar as possible to a filtered version of a Dirac delta. Therefore, the weighting function $h\left(\rho, z^{\prime}\right)$ must be chosen such as to make $K\left(w, z^{\prime}\right)=1$, that is

$$
h\left(\rho, z^{\prime}\right)=\frac{1}{\left.\left|G\left(\rho, z^{\prime}\right)\right|^{2} \frac{\partial \rho}{\partial w}\right|_{z=z^{\prime}}}
$$


In particular, since $w\left(z, z^{\prime}, \rho\right)$ at $z=z^{\prime}$ is

$$
w\left(z^{\prime}, \rho\right)=\frac{\partial \phi(\rho, z)}{\partial z}=\frac{k z}{R}
$$

then

$$
\left.\frac{\partial \rho}{\partial w}\right|_{z=z^{\prime}}=\frac{\partial w^{-1}}{\partial \rho}=-\frac{R^{3}}{k z \rho}
$$

Hence, by choosing

$$
h\left(\rho, z^{\prime}\right)=\frac{k z \rho}{R^{3}\left|G\left(\rho, z^{\prime}\right)\right|^{2}}
$$

the $p s f_{h}\left(z, z^{\prime}\right)$ becomes

$$
\operatorname{ps} f_{h}\left(z, z^{\prime}\right)=e^{-j\left[\gamma(z)-\gamma\left(z^{\prime}\right)\right]} \operatorname{sinc}\left[\Delta w\left(z, z^{\prime}\right)\left(z-z^{\prime}\right)\right] \Delta w\left(z^{\prime}\right)
$$

Note that the non oscillating term in (26) $\Delta w\left(z, z^{\prime}\right)$ has been approximated with its values at $z=z^{\prime}$. This perfectly accords to the leading term approximation exploited in (19).

Now, by applying Rayleigh's criterion, the resolution can be quantified as the halfwidth of the point-spread function main lobe. For simplicity, we introduce the warping transformation $\xi(z)$, such as $\Delta w\left(z, z^{\prime}\right)\left(z-z^{\prime}\right)=\xi(z)-\xi\left(z^{\prime}\right)[26,27]$ whose expression in terms of $z$ is

$$
\xi=\frac{k}{2}\left[\sqrt{a^{2}+z^{2}}-z\right]
$$

Let $\Delta \xi$ be the distance between the maximum and the first zero of $p s f_{h}$. It holds that

$$
\Delta \xi=\pi
$$

Returning to variable $z$ by (27), the equation (28) can be rewritten as

$$
\frac{1}{\lambda}\left[\sqrt{a^{2}+z^{2}}-z-\sqrt{a^{2}+(z+\Delta z)^{2}}+z+\Delta z\right]=1
$$

where $z$ and $z+\Delta z$ are the locations in $z$ of the maximum and the first null of $p s f_{h}$.

By solving (29), we obtain

$$
\Delta z=\frac{\lambda}{2}\left[\frac{\lambda-2 \sqrt{a^{2}+z^{2}}}{z+\lambda-\sqrt{a^{2}+z^{2}}}\right]
$$

Note that the transformation in (27) is very similar to the one proposed in [14] meaning that (30) works within the region to which the pseudo-Fresnel approximation applies. As can be appreciated the on-axis resolution $\Delta z$ is not constant along with the observation domain but it depends on the point location $z$. The $\Delta z$ becomes low (resolution improves) close to the aperture and increases as $z$ moves far from it (see Figure 3). This means that $E_{i}$ will not approximate $E_{o}$ with the same level of accuracy along the $z$ axis. In particular, the approximation will be better for the points closer to the aperture while degrades far from it. Such a non-uniform behavior of resolution has already been observed in many recent papers [2,3] and it is distinctive to near non-reactive field zone. 


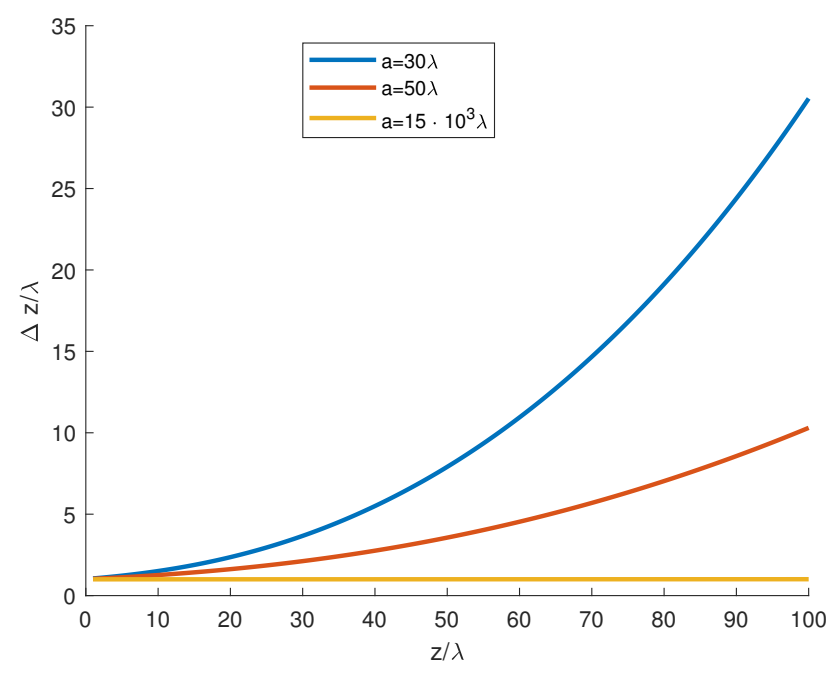

Figure 3. Resolution returned by (30) for different values of aperture radius.

The resolution formula (30) makes clear also the role of radius aperture. As can be appreciated in Figure 3, resolution improves when $a$ increases. Finally, it is seen that coherently with classical plane-wave spectrum arguments, as $a$ approaches $\infty \Delta z$ tends to be uniform along with the observation domain and equal to $\lambda$. Note that the latter is the minimum value of resolution that can be also achieved as $z^{\prime}$ approaches 0 . This is clear because we only considered $r \geq \lambda$ so that evanescent waves do not play any role.

Moreover, since the latter is the only assumption made on the distance between the aperture and observation domain, (30) highlights the impact of aperture geometrical parameters on-axis resolution for distances that are below the Fresnel zone. Hence, such a formula includes all the resolution estimations derived in the literature under more restrictive conditions. For example under the Fresnel approximation, that is, $z>>z_{F}=$ $\left[\frac{\pi a^{4}}{4 \lambda}\right]^{\frac{1}{3}}[28]$ the following holds

$$
z \sqrt{1+\left[\frac{a}{z}\right]^{2}} \approx z\left[1+\frac{a^{2}}{2 z^{2}}\right]
$$

and the Equation (29) returns

$$
\frac{a^{2}}{2 \lambda}\left[\frac{1}{z}-\frac{1}{z+\Delta z}\right]=1
$$

Such formula is in agreement with the one derived in [15].

Note that in the latter paper (32) has been derived by estimating the singular system of the operator $\mathcal{A}$ in closed form. Here, we are not able to do that, but we exploit an alternative approach that allows obtaining an approximation of psf evaluated in terms of eigenfunctions $v_{n}$. The goodness of such an approximation will be verified numerically in the next section. However, the fact that (30) under the Fresnel approximation returns the resolution formula derived in [15] justifies the expectation that (26) will be in good agreement with (8).

\section{Numerical Analysis}

In this section the point spread-functions $p s f$ and $p s f_{h}$ given by (26) are compared. Two apertures with different radii are considered. At first, we set $a=30 \lambda$. Note that for such an aperture the Fresnel distance is $z_{F}=86 \lambda$.

Accordingly, in Figures 4 and 5, the magnitudes of the normalized point spread functions are shown for distances below and comparable to $z_{F}$. In particular, in Figure 4 the observation domain ranges from $z_{1}=\lambda$ to $z_{2}=30 \lambda$, while in Figure $5 z_{1}=15 \lambda$ and $z_{2}=200 \lambda$. The blue lines refer to $p s f$, while the dotted red lines to $p s f_{h}$ for different values of $z^{\prime}$ (in Figure $4 z^{\prime}=\{5 \lambda, 15 \lambda, 25 \lambda\}$, in Figure $5 z^{\prime}=\{60 \lambda, 80 \lambda, 120 \lambda\}$ ). As can be seen, 
the two curves are in good agreement for both the considered cases; accordingly, (29) can be exploited to estimate the resolution. In particular, as expected, the main lobe of the point spread function becomes larger as the $z^{\prime}$ increases.

The results concerning the second very large aperture $a=15 \times 10^{3} \lambda$ are shown in Figure 6. Additionally for this case, the figure shows the magnitudes of the normalized point spread functions for distances below $z_{F}=3.410^{5} \lambda$. In particular, the observation domain ranges from $z_{1}=2 \lambda$ to $z_{2}=50 \lambda$. Again, the blue and red lines overlap very well and as expected, since $a>>\lambda$, the resolution appears uniform along the observation domain and equal to $\lambda$.
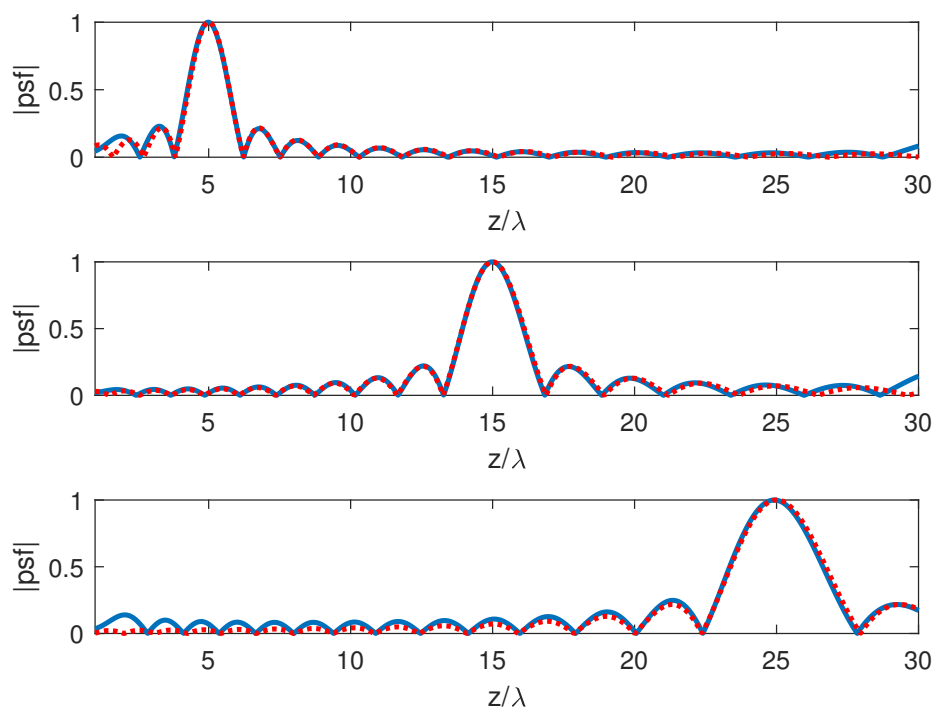

Figure 4. Magnitude of the point spread function for $a=30 \lambda, z_{1}=\lambda$ and $z_{2}=30 \lambda$. The blue line is $p s f$, while the dotted red line refers to $p s f_{h}$. In the top panel $z^{\prime}$ is $5 \lambda$, in the middle $15 \lambda$ and in the bottom $25 \lambda$.
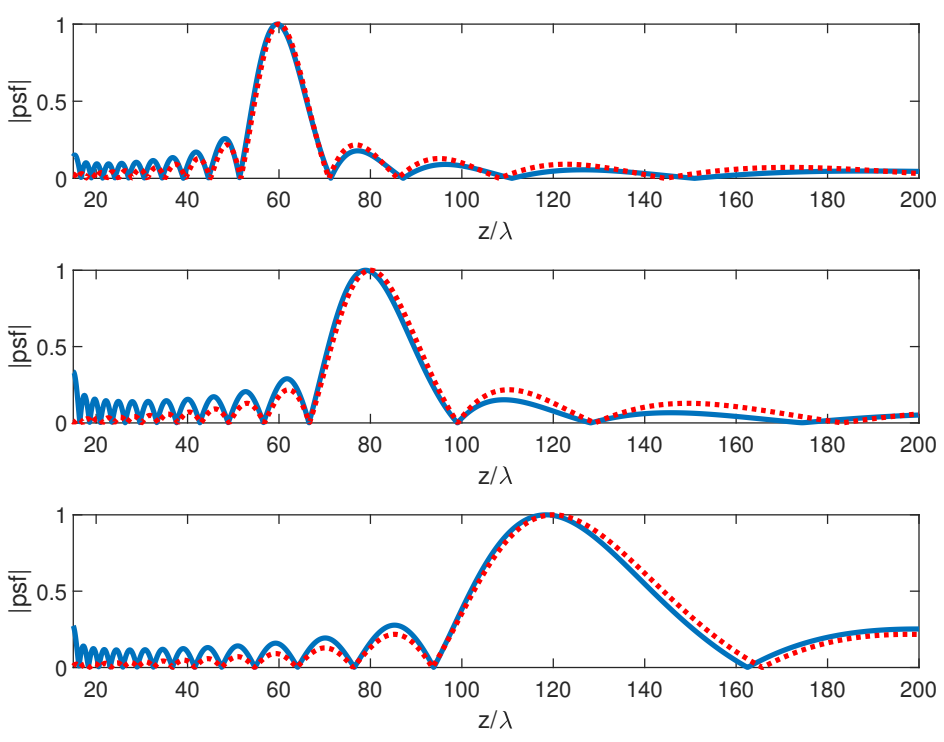

Figure 5. Magnitude of the point spread function for $a=30 \lambda, z_{1}=15 \lambda$ and $z_{2}=200 \lambda$. The blue line is $p s f$, while the dotted red line refers to $p s f_{h}$. In the top panel $z^{\prime}$ is $60 \lambda$, in the middle $80 \lambda$ and in the bottom $120 \lambda$. 

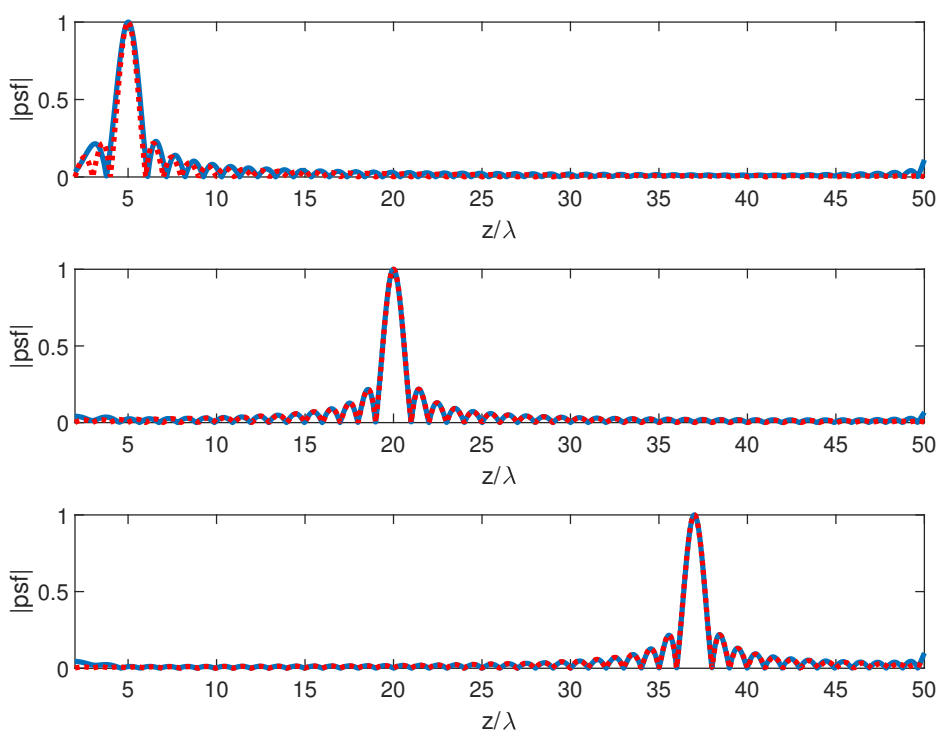

Figure 6. Magnitude of the point spread function for $a=15 \times 10^{3} \lambda, z_{1}=2 \lambda$ and $z_{2}=50 \lambda$. The blue line is $p s f$, while the dotted red line refers to $p s f_{h}$. In top panel $z^{\prime}$ is $5 \lambda$, in the middle $20 \lambda$ and in the bottom $37 \lambda$.

\section{Number of Degree of Freedom Estimation}

As is well known, and as we mentioned above, the NDF is a very important parameter. Resolution limits arise because $N$ is a bounded number. In the theory of communication mode, such a number gives a measure of the maximal number of communication modes that are "significantly" connected [9]. Hence, it allows obtaining an estimation of information transmitted by the aperture $A$ to the observation domain. In the framework of antenna synthesis, it identifies the number of independent radiation patterns that the antenna can radiate [11] or, in general, the dimension of the radiation operator Range [29]. In array and grid scatterer diagnostics, it suggests the maximum number of elements that can be determined [10,30]. Moreover, $N$ represents the number of independent data [31], a very relevant parameter that allows controlling the occurrence of local minima in Phase retrieval problem [32]. It is estimated as the number of more "significant" singular values of the operator describing the problem under concern. However, when the singular values are not known in closed form, as for the case at hand, different approaches can be followed to get an estimation of it. For example, in optics, it is common to estimate the NDF as the number of distinguishable spots that can be realized within a given observation aperture [33]. By following the same reasoning, here, the NDF is estimated by counting how many nonoverlapping point spread function main beams are required to fill the observation domain. Since the observation interval $\left[z_{1}, z_{2}\right]$ maps into a new one in $\xi$ variable $\left[\xi_{\min }, \xi_{\text {max }}\right]$, this approach can be conveniently worked out in the $\xi$ domain, where the main beam of the point spread function is constant and given by (28). Accordingly, we obtain

$$
N D F=\frac{\xi_{\text {max }}-\xi_{\text {min }}}{\Delta \xi}
$$

and finally, using (27),

$$
N D F=\frac{1}{\lambda}\left(\sqrt{a^{2}+z_{1}^{2}}-\sqrt{a^{2}+z_{2}^{2}}+z_{2}-z_{1}\right)
$$

Of course, (34) does not in general return an integer value. Therefore, in the following, the NDF is estimated as the lowest integer which is greater than (34). In Figure 2, the normalized singular values of $\mathcal{A}$ are shown in $\mathrm{dB}$. In both cases, observation domain ranges from $z_{1}=2 \lambda$ to $z_{2}=50 \lambda$, while on left the radius $a$ is set to $30 \lambda$, on right to $15 \times 10^{3} \lambda$. According to such geometrical parameters, (34) returns 47 and 19 that, as it 
can be appreciated from the figure, represent the singular value indexes at which the knee occurs.

\section{Conclusions}

In this paper, the on-axis resolution of a circular aperture in creating a desired field profile has been studied. In particular, its expression in terms of aperture geometrical parameters is provided. Our investigation is directly connected to that in [15]. However, here the derived resolution formula extends the one in [15] because it covers configurations that are within the near non-reactive approximation. For the considered geometry, the singular values decomposition of radiation operator cannot be derived in closed form so in order to evaluate the point-spread function, an alternative approach has been proposed. This is based on the observation that to find the field over the aperture ensuring a target field profile on the observation domain requires solving an inverse problem. Accordingly, by solving the latter with the weighted adjoint inversion method and introducing some manipulations, an analytical expression for the point spread function in terms of geometrical parameters is derived. The obtained estimation highlights the role played by the configuration geometrical parameters. Moreover, the resolution is non-uniform along the $z$ axis meaning that while near the aperture smaller details can be retrieved, as the distance increases this ability degrades. This behavior is emphasized as the aperture radius decreases, on the contrary, the resolution appears uniform for a very large aperture.

Funding: This research was funded by the Universitá della Campania Luigi Vanvitelli by funding, through Programma V:ALERE 2020, the project Efficient Probe pOsitioning for Near-fleld Measurement technIques (EPONIMI) CUP: B66J20000680005.

Acknowledgments: The author kindly thanks Raffaele Solimene for his suggestions.

Conflicts of Interest: The authors declare no conflict of interest.

\section{References}

1. den Dekker, A.J.; Bos, A.V. Resolution: A survey. J. Opt. Soc. Am. A 1997, 14, 547-557. [CrossRef]

2. Maisto, M.A.; Solimene, R.; Pierri, R. Resolution limits in inverse source problem for strip currents not in Fresnel zone. J. Opt. Soc. Am. A 2019, 36, 826-833. [CrossRef] [PubMed]

3. Maisto, M.A.; Solimene, R.; Pierri, R. Depth resolution in strip current reconstructions in near non-reactive zone. J. Opt. Soc. Am. A 2019, 36, 975-982. [CrossRef] [PubMed]

4. Pierri, R.; Liseno, A.; Soldovieri, F.; Solimene, R. In-depth resolution for a strip source in the Fresnel zone. J. Opt. Soc. Am. A 2001, 18, 352-359. [CrossRef] [PubMed]

5. Maisto, M.A.; Solimene, R.; Pierri, R. Transverse Resolution in Microwave Imaging for Strip Objects Buried in a Half-Space Medium. Prog. Electromagn. Res. 2020, 88, 145-157. [CrossRef]

6. Thaning, A.; Martinsson, P.; Karelin, M.; Friberg, A.T. Limits of diffractive optics by communication modes. J. Opt. A Pure Appl. Opt. 2003, 5, 153. [CrossRef]

7. Miller, D.A.B. Communicating with waves between volumes: Evaluating orthogonal spatial channels and limits on coupling strengths. Appl. Opt. 2000, 39, 1681-1699. [CrossRef]

8. Hille, E.; Tamarkin, J.D. On the characteristic values of linear integral equations. Proc. Natl. Acad. Sci. USA 1928, 14, 911-914. [CrossRef]

9. Piestun, R.; Miller, D.A.B. Electromagnetic degrees of freedom of an optical system. J. Opt. Soc. Am. A 2000, 17, 892-902. [CrossRef]

10. Leone, G.; Maisto, M.A.; Pierri, R. Inverse Source of Circumference Geometries: SVD Investigation Based on Fourier Analysis. Prog. Electromagn. Res. 2018, 76, 217-230. [CrossRef]

11. Leone, G.; Munno, F.; Pierri, R. Radiation properties of conformal antennas: The elliptical source. Electronics 2019,8 , 531. [CrossRef]

12. Burch, D.S. Fresnel diffraction by a circular aperture. Am. J. Phys. 1985, 53, 255-260. [CrossRef]

13. Overfelt, P.L.; Kenney, C.S. Comparison of the propagation characteristics of Bessel, Bessel-Gauss, and Gaussian beams diffracted by a circular aperture. J. Opt. Soc. Am. A 1991, 8, 732-745. [CrossRef]

14. Sheppard, C.J.R.; Hrynevych, M. Diffraction by a circular aperture: A generalization of Fresnel diffraction theory. J. Opt. Soc. Am. A 1992, 9, 274-281. [CrossRef]

15. Burvall, A.; Martinsson, P.; Friberg, A.T. Communication modes applied to axicons. Opt. Express 2004, 12, 377-383. [CrossRef] 
16. Leone, G. Source Geometry Optimization for Hemispherical Radiation Pattern Coverage. IEEE Trans. Antennas Propag. 2016, 64, 2033-2038. [CrossRef]

17. Wang, Y.; Yan, S.; Friberg, A.T.; Kuebel, D.; Visser, T.D. Electromagnetic diffraction theory of refractive axicon lenses. J. Opt. Soc. Am. A 2017, 34, 1201-1211. [CrossRef]

18. Dell'Aversano, A.; Leone, G.; Ciaramaglia, F.; Solimene, R. A Strategy for Reconstructing Simple Shapes From Undersampled Backscattered Data. IEEE Geosci. Remote Sens. Lett. 2016, 13, 1757-1761. [CrossRef]

19. Bertero, M.; Boccacci, P. Introduction to Inverse Problems in Imaging; Institute of Physics Publishing: Bristol, UK, 1998. [CrossRef]

20. Cheney, M.; Bonneau, R.J. Imaging that exploits multipath scattering from point scatterers. Inverse Probl. 2004, $20,1691-1711$.

21. Horiuchi, K. Sampling principle for continuos signals with time-varying bands. Inf. Control 1968, 13, 53-61. [CrossRef]

22. Wei, D.; Oppenheim, A.V. Sampling Based on Local Bandwidth. In Proceedings of the Conference Record of the Forty-First Asilomar Conference on Signals, Systems and Computers, Pacific Grove, CA, USA, 4-7 November 2007. [CrossRef]

23. Maisto, M.A.; Solimene, R.; Pierri, R. Sampling approach for singular system computation of a radiation operator J. Opt. Soc. Am. A 2019, 36, 353-361.

24. Soumekh, M. Depth-focused interior echo imaging. IEEE Trans. Image Process. 1999, 8, 1608-1618.

25. Leone, G.; Soldovieri, F. Analysis of the Distorted Born Approximation for Subsurface Reconstruction: Truncation and Uncertainties Effects. IEEE Trans. Geosci. Remote Sens. 2003, 41, 66-74. [CrossRef]

26. Clark, J.J.; Palmer, M.R.; Lawrence, P.D. A transformation method for the reconstruction of functions from nonuniformly spaced samples. IEEE Trans. Acoust. Speech Signal Process. 1985, 33, 1151-1165. [CrossRef]

27. Cochran, D.; Clark, J.J. On the sampling and reconstruction of time warped band-limited signals. In Proceedings of the International Conference on Acoustics, Speech, and Signal Processing, Albuquerque, NM, USA, 3-6 April 1990. [CrossRef]

28. Goodman, J.W. Introduction to Fourier Optics, 2nd ed.; McGraw-Hill: Singapore, 1996.

29. Maisto, M.A.; Solimene, R.; Pierri, R. Valid angle criterion and radiation pattern estimation via singular value decomposition for planar scanning. IET Microw. Antennas Propag. 2019, 13, 2342-2348.

30. Brancaccio, A.; Solimene, R. Fault detection in dielectric grid scatterers. Opt. Express 2015, 23, 8200-8215. [CrossRef]

31. Brancaccio, A.; Dell'Aversano, A.; Leone, G.; Solimene, R. Subsurface Detection of Shallow Targets by Undersampled Multifrequency Data and a Non-Cooperative Source. Appl. Sci. 2019, 9, 5383. [CrossRef] [PubMed]

32. Moretta, R.; Pierri, R. Performance of Phase Retrieval via Phaselift and Quadratic Inversion in Circular Scanning Case. IEEE Trans. Antennas Propag. 2019, 67, 7528-7537. [CrossRef]

33. Gabor, D. Light and information. In Progress in Optics; Wolf, E., Ed.; North-Holland: Amsterdam, The Netherlands, 1961; Volume 1, pp. 109-153. [CrossRef] 